\title{
Relação entre Gênero e Orientação Sexual a partir da Perspectiva Evolucionista ${ }^{1}$
}

\author{
Aline Beckmann Menezes ${ }^{2}$ \\ Regina Célia Souza Brito \\ Alda Loureiro Henriques \\ Universidade Federal do Pará
}

\begin{abstract}
RESUMO - Este estudo trata da relação entre gênero e orientação sexual a partir da perspectiva interacionista da Psicologia Evolucionista e da análise de diferentes elementos da sexualidade humana. Procurou-se discutir a literatura existente sobre os conceitos de gênero e de orientação sexual, com base nos quatro porquês da Etologia. Propõe-se a existência de múltiplas origens para a orientação sexual, sendo uma delas relacionada aos padrões típicos de gênero e à identidade de gênero. Isso levaria à identificação com indivíduos do mesmo sexo ou do sexo oposto e, consequentemente, à atração pelo grupo diferente daquele com o qual se desenvolveu a identificação. Essa perspectiva integra pré-disposições biológicas à análise de influências culturais, compreendendo, como complementares, vertentes teóricas usualmente tidas como contraditórias.
\end{abstract}

Palavras-chave: orientação sexual; gênero; identidade; Psicologia Evolucionista.

\section{Relation between Gender and Sexual Orientation from the Evolutionary Approach}

\begin{abstract}
This study addresses the relationship between gender and sexual orientation from the interacionist perspective of Evolutionary Psychology and the analysis of various aspects of human sexuality. This article aimed to discuss existing literature about gender and sexual orientation, based on four questions of Ethology. It's proposed that sexual orientation has multiple origins, and that one of those is related to typical patterns of gender and gender identity. This would lead to identification with same-sex or opposite-sex individuals, and hence, to the attraction for the group different from the one to which identification was established. This approach integrates biological pre-dispositions to the analysis of cultural influences, understanding, as complementary, theoretical views that are usually stated as contradictory.
\end{abstract}

Keywords: sexual orientation; gender; identity; Evolutionary Psychology.

A Psicologia Evolucionista propõe o estudo de mecanismos psicológicos que foram selecionados durante a evolução da espécie humana, de modo a afetarem os comportamentos observados atualmente (Tooby \& Cosmides, 2000). Essa proposta está fundamentada no paradigma darwinista de seleção das espécies e compreende que, no Ambiente de Adaptação Evolutiva (AAE), desenvolveram-se mecanismos psicológicos (relativos ao funcionamento neurológico e fisiológico do organismo) que favoreceram o rápido aprendizado de determinados padrões devido à suscetibilidade a determinadas estimulações ambientais específicas - aquelas que fossem importantes para sobrevivência e reprodução.

Considerando essa perspectiva, diversos estudos têm sido realizados buscando analisar quais mecanismos foram selecionados e como ocorre o funcionamento dos mesmos no contexto atual (Tooby \& Cosmides, 2000). Neste artigo, pretende-se discutir a importância do dimorfismo sexual humano e sua relação com a orientação sexual. Para tal, fazse necessário discutir a forma como serão abordados aqui alguns conceitos básicos.

1 Agradecemos o apoio e colaboração dos membros do Grupo de Estudos Avançados em Psicologia Evolucionista da UFPA (GEAPE). Este trabalho foi desenvolvido como parte da Tese de Doutorado da primeira autora, sob orientação da segunda.

2 Endereço para correspondência: Tv. Benjamin Constant 1308, Apto. 42 , Nazaré. Belém, PA. CEP 66035-060. E-mail: alinebcm@gmail.com.

\section{Orientação Sexual}

O estudo da orientação sexual humana tem sido desenvolvido há muito tempo, sob diferentes perspectivas. Em geral, as pesquisas partem de dois pressupostos básicos, a saber: (1) a heterossexualidade não requer explicação, de modo que basta ser estudada a homossexualidade (Bem, 1996; Birke, 1981; LeVay, 1996; Trevisan, 2002); (2) a orientação sexual (seja ela homo ou heterossexual) caracteriza-se como fenômeno único, de modo que haveria uma resposta única para explicar o desenvolvimento da mesma (Bem, 2000). Tais pressupostos permeiam a amostragem, o método e a análise dos resultados das pesquisas desenvolvidas, de modo que não há hoje na literatura acadêmica uma posição consolidada acerca do assunto (Menezes, 2005).

Primeiramente, o foco dos estudos que buscam uma compreensão do comportamento homossexual implica em duas principais conclusões: (1) o comportamento homossexual seria uma anomalia, logo requereria um estudo específico como os existentes acerca de patologias; (2) o comportamento heterossexual seria natural, por sua explícita função reprodutiva, dispensando maiores investigações.

Tal posicionamento reflete um viés dos pesquisadores (indicando preconceito e atribuição de valores diferenciados aos padrões hetero e homossexual) e atribui naturalidade à heterossexualidade em função do valor reprodutivo, ignoran- 
do outras funções da prática sexual. Menezes e Brito (2007) discutem as diversas funções da prática sexual e da evolução do prazer (como o estabelecimento de vínculos afetivos), enfatizando a plasticidade sexual humana e a sua importância para o estabelecimento de vínculos entre parceiros (fundamental ao cuidado parental). Assim, as autoras propõem a possibilidade da espécie humana não ter evoluído com uma orientação sexual pré-definida, mas com seus membros sendo aptos a múltiplas estimulações sexuais que possibilitariam, como subproduto, o desenvolvimento tanto da hetero quanto da homossexualidade.

Pode-se questionar, portanto, se há fundamentação para a afirmação de que a heterossexualidade seria natural e, consequentemente, já estaria explicada (proposta defendida por muitos autores, desde o século XIX, tais como Krafft-Ebbing, conforme citado por Feldman, 2003, e mais recentemente, Gooren, 2006). Para tal, seria necessário identificar os mecanismos de limitação da atração sexual, isto é, os elementos genéticos e/ou fisiológicos que seriam responsáveis pela identificação de indivíduos do sexo oposto e pela limitação da responsividade sexual àquelas situações em que a estimulação sexual é gerada unicamente por esses indivíduos. Tais mecanismos restritivos implicariam em um elevado custo evolutivo (Lorenz, 1965/1986) e não são condizentes com a ampla suscetibilidade à estimulação sexual observada no cotidiano (Kinsey, Pomeroy \& Martin, 1948).

$\mathrm{O}$ segundo pressuposto supracitado refere-se à compreensão da orientação sexual como um fenômeno unifatorial, o que se reflete na busca por uma única teoria sobre o seu desenvolvimento (Menezes, 2005). Como Kinsey e cols. (1948) já haviam demonstrado, a orientação sexual pode ser observada a partir de um continuum de sete possíveis classificações: (1) Exclusivamente heterossexual; (2) Predominantemente heterossexual com episódios raros de homossexualidade; (3) Predominantemente heterossexual com múltiplos episódios de homossexualidade; (4) Tanto heterossexual quanto homossexual; (5) Predominantemente homossexual com múltiplos episódios de heterossexualidade; (6) Predominantemente homossexual com episódios raros de heterossexualidade; (7) Exclusivamente homossexual.

Além disso, muitos autores indicam que a classificação pode variar de acordo com as dimensões investigadas da orientação sexual (ver revisão dessas classificações em Menezes, 2005), como, por exemplo, atração sexual, fantasias sexuais, práticas sexuais, identidade etc.

Pode-se, ainda, defender que dois padrões comportamentais topograficamente similares podem resultar de diferentes histórias de aprendizagem, obtendo assim diferentes funções. Isto é, a observação de comportamentos públicos similares entre dois indivíduos não significa necessariamente que o processo de aprendizagem desses padrões tenha sido o mesmo. A inferência de uma única explicação para um fenômeno (neste caso, a orientação sexual) com base apenas na similaridade de seu produto (no caso, o sexo do parceiro) seria uma supersimplificação, tanto do fenômeno em si (já que não é apenas o sexo do parceiro que leva um indivíduo a se considerar homossexual), quanto dos diferentes processos de evolução e aprendizagem de padrões comportamentais.

Assim, questiona-se o porquê das múltiplas manifestações da orientação sexual - cujas topografias de resposta são ex- tremamente variáveis - serem consideradas como resultantes de uma mesma história evolutiva (onto e filogeneticamente). Pode-se interpretar as dissonâncias e inconsistências existentes interpesquisadores como possível fruto de serem objetos de estudo distintos, erroneamente agrupados sob uma única categoria comportamental.

Dessa forma, o primeiro ponto a ser defendido aqui é a importância do estudo da orientação sexual humana como fenômeno complexo, constituído de diferentes padrões e funções.

A caracterização da orientação sexual como fenômeno complexo incorre, entre outras coisas, na necessidade de rompimento com a tradicional dicotomia inato e aprendido. Assim, a perspectiva interacionista faz-se fundamental para articular os diferentes resultados existentes na literatura, possibilitando a interpretação de que, ao invés de contraditórios, estes seriam complementares.

Nessa direção de complementaridade - ou mesmo indissociabilidade - Bussab e Ribeiro (1998) discutem como a espécie humana é "biologicamente cultural". Dessa forma, os autores desenvolvem a noção de que o confronto entre biologia e cultura seria, na verdade, inconsistente, já que a estrutura biológica do ser humano evoluiu de modo a necessitar da inserção cultural e, paralelamente, a cultura se estabelece em consonância com suscetibilidades orgânicas específicas (ver ainda Skinner, 1975).

Pode-se compreender que padrões comportamentais complexos são, inexoravelmente, frutos da interinfluência biológica e cultural, em um ciclo contínuo de modificações individuais. Defende-se aqui, portanto que se pode considerar que a orientação sexual não seria resultante diretamente nem de fatores biológicos nem de culturais, mas que suas múltiplas formas de manifestação decorreriam de diferentes interações entre esses elementos e, consequentemente, de diferentes percursos de desenvolvimento ontogenético.

Dentre as diversas linhas de investigação sobre a determinação da orientação sexual humana, as pesquisas que envolvem padrões típicos de gênero (como diferentes preferências por brincadeiras ou habilidades na realização de diferentes tarefas, por exemplo) podem ser consideradas como exemplificação da proposta de compreensão apresentada acima (e.g., Bem, 1996; Van Wyk \& Geist, 1984). Isso porque os padrões típicos de gênero são desenvolvidos a partir da constituição biológica e de influências culturais.

\section{Sexo, Gênero e Dimorfismo Sexual}

Tradicionalmente, os conceitos de sexo e de gênero tendem a ser diferenciados, na literatura, a partir da dicotomia biologia e cultura. Assim, características anatômicas seriam a base para a definição de sexo enquanto que características socioculturais determinariam o gênero. Por outro lado, perspectivas de fundamentação evolutiva tendem a refutar a diferenciação entre os conceitos de sexo e gênero, a partir da argumentação de que comportamentos típicos de um gênero estariam invariavelmente associados ao sexo (isto é, macho/ masculino e fêmea/feminino seriam relações indissociáveis). É, ainda, argumentado que a inter-relação entre biologia e cultura se daria em todos os padrões comportamentais 
humanos, de forma que considerar o gênero como cultural e sexo como biológico não seria um critério suficiente para justificar a separação entre os conceitos de gênero e sexo.

Todavia, a distinção entre tais conceitos é importante, mesmo na perspectiva aqui adotada. Padrões tipicamente masculinos são mais comuns em homens que em mulheres, e vice-versa, mas há um grande número de indivíduos (independentemente da orientação sexual) geneticamente masculinos (isto é, possuem cromossomos XY) que apresentam comportamentos tipicamente femininos. Esse fato demonstra que a compreensão dos padrões típicos de gênero não pode se restringir aos cromossomos sexuais, devendo abranger elementos do desenvolvimento bio-fisiológico bem como socioculturais.

Assim, propõe-se a adoção do termo "gênero" para se referir a padrões comportamentais tipicamente masculinos e femininos (de acordo com a maior frequência de emissão dos mesmos entre homens e mulheres), "sexo" para a diferenciação genética e morfológica dos organismos em homens e mulheres (tendo a intersexualidade como um desafio teórico de enquadramento) e, por fim, "identidade de gênero" para a forma como um indivíduo se percebe e se classifica como masculino ou feminino.

Exemplos de padrões típicos de gênero são múltipos, sendo alguns associados à constituição cerebral, como a agressividade, a força física, o comportamento parental etc. (Alexander, 2003; Gadpaille, 1980; Pinker, 2004). Hansen, Macarini, Martins, Wanderlind e Vieira (2007) e Silva e cols. (2006) descrevem a preferência das meninas por brincadeiras sociointerativas, com temáticas domésticas e familiares, bem como a valorização da harmonia e da cooperação. Meninos, por outro lado, apresentam predileção por brincadeiras de contingências físicas, com temas fantásticos ou com uso predominante de ferramentas.

Lippa (2005), por sua vez, destaca diferenças na escolha ocupacional, havendo uma predileção por ocupações realistas, relacionadas a coisas e informações, por pessoas mais masculinas e uma predileção por ocupações sociais ou artísticas, relacionadas a pessoas e ideias, por pessoas mais femininas. Para esse autor, a masculinidade e a feminilidade comporiam uma dimensão bipolar contínua (M-F), havendo indivíduos de ambos os sexos situados em diferentes pontos da mesma. Lippa defende, ainda, a existência de diferenças universais que delimitam a amplitude da dimensão, mas que a influência cultural em cada contexto específico pode decorrer em uma classificação diferenciada. Isso significa dizer que há uma predisposição para comportar-se de determinadas formas de acordo com o sexo; contudo, o conceito de gênero também recebe influências culturais que estabelecem critérios variados do que seria considerado comportamento masculino ou feminino em cada grupo.

Essa proposta é muito importante para a discussão feita neste estudo. A relação entre pressões evolutivas e o estabelecimento de práticas culturais tem sido discutida, tendo vários mecanismos psicológicos sob análise (ver o conceito de 'cultura evocada' em Gangestad, Haselton \& Buss, 2006). Pode-se propor, portanto, que evolutivamente foi importante que indivíduos do sexo masculino e do feminino tivessem diferentes mecanismos selecionados, privilegiando a especialização de tarefas segundo demandas específicas (como cuidado com a prole, a caça etc.). Tais diferenças tornam alguns comportamentos mais prováveis do que outros, mas não impossibilitam a variabilidade comportamental, já que esta também é importante para a adaptabilidade a contextos diversos. Assim, práticas culturais emergem a partir das demandas de cada sexo, intensificando ou reduzindo o dimorfismo sexual segundo o contexto.

O dimorfismo sexual pode ser definido como as diferenças inerentes a cada sexo no que se refere às predisposições orgânicas. Por exemplo, habilidades de processamento espacial, concentração, empatia etc. têm sido demonstradas como sexualmente dimórficas na espécie humana, de modo que o sexo é um forte preditor de como tal habilidade específica pode ser desenvolvida (e.g., Haussmann, 2005; Lalumière, Blanchard \& Zucker, 2000; Wegesin, 1998). Kraemer e cols. (2009) relatam que taxas elevadas de testosterona no desenvolvimento pré-natal estariam associadas à masculinização do feto, incluindo aspectos comportamentais observáveis ao longo do desenvolvimento posterior. Estes autores investigaram (a partir da medida 2D:4D) os efeitos das taxas pré-natais de testosterona sobre o transtorno de identidade de gênero e encontraram correlação negativa em participantes do sexo masculino e positiva nos do sexo feminino, corroborando a hipótese de que os padrões típicos de gênero estariam relacionados à produção hormonal.

Alexander, Wilcox e Woods (2009), por sua vez, encontraram que o interesse em brinquedos como estímulos visuais (medido a partir da frequência e duração do olhar direcionado a bonecas ou caminhões) é sexualmente dimórfico. Enquanto meninos olham mais para os caminhões, mas com diferença não significativa, meninas olham significativamente mais para bonecas $(\mathrm{d}=1,27, \mathrm{p}<0,01)$. Os autores discordam de hipóteses sociais para explicar tal diferença, já que a mesma pode ser identificada desde os 9 meses de idade, isto é, antes mesmo de ser desenvolvida uma história de interação com aqueles brinquedos em particular.

O dimorfismo sexual também é encontrado em outras espécies, especialmente em primatas, com diferenças similares às humanas. Por exemplo, Alexander e Hines (2002) e Hassett, Siebert e Wallen (2008) estudaram a preferência por brinquedos em primatas não humanos do Velho Mundo e observaram um paralelo muito forte com os dados obtidos em pesquisas com brincadeiras sexualmente tipificadas em infantes da espécie humana. Alexander e Hines observaram que os machos tendiam a passar mais tempo interagindo com bolas e carros do que as fêmeas, enquanto as fêmeas detinham-se mais prolongadamente com bonecas e panelas do que os machos. O tempo dedicado a objetos classificados como neutros (como um livro acolchoado) foi o mesmo por machos e fêmeas. Hassett, Siebert e Wallen, por sua vez, criaram situações de escolha contrapondo brinquedos de movimento (i.e. com rodas) e outros de apertar (i.e. pelúcia), havendo mais escolhas pelos de movimentos entre os machos, mas pouca diferença entre as fêmeas. Os dados dessas pesquisas não são conclusivos, mas a ausência de atribuição de um significado cultural para os brinquedos utilizados pelos sujeitos é um fator relevante para a identificação de diferenças de gênero que prescindem da mediação cultural.

Outro conceito que necessita ser compreendido é o de identidade sexual. Alguns autores têm discutido como pa- 
drões típicos de gênero possuem um importante papel para a construção da identidade sexual, entretanto esses conceitos não são sinônimos (e.g., Baker, 1980; Minton \& McDonald, 1984). Como já foi dito anteriormente, a identidade sexual refere-se à forma como um indivíduo se percebe, como membro do grupo formado por pessoas do sexo masculino ou do feminino. Gooren (2006) discute a importância desse sentimento de pertencimento, resultante da identidade sexual, para a construção da identidade como um todo.

Um bom exemplo para compreender essas distinções apresentadas é a linha de estudos com portadores de hiperplasia adrenal congênita $(\mathrm{CAH})$, síndrome na qual os portadores possuem cromossomos sexuais, gônadas e sistema reprodutor interno femininos, hormônios masculinos e sistema reprodutor externo ambíguo. Esses indivíduos demonstram que a criação como meninas implica no desenvolvimento de identidade de gênero feminina, mas na manutenção de comportamentos tipicamente masculinos (e.g., Alexander \& Hines, 2002; Almeida, 2007; Baker, 1980; Le Vay, 1996; Ricketts, 1984; Sardinha, 2007). Poder-se-ia dizer, assim, que são pessoas do sexo feminino, com padrões típicos do gênero masculino (remetendo, inclusive à dimensão $\mathrm{M}-\mathrm{F}$ proposta por Lippa, 2005), mas com identidade de gênero feminina. Pode-se esperar, ainda, que o dimorfismo sexual seja ambíguo, em função dos hormônios masculinos - hipótese esta que precisaria ainda ser mais investigada.

Hassett e cols. (2008) defendem que os tipos de atividades preferidas por meninos e meninas teriam forte influência biológica, mas a sua forma de manifestação e os brinquedos utilizados seriam derivados do contexto e da atribuição de significado social aos objetos e situações, isto é, os brinquedos possibilitariam a expressão de um modo evolutivamente selecionado de se relacionar com objetos.

Sintetizando esse item, pode-se compreender que as diferenças de gênero possuem uma função evolutiva muito importante. O papel desenvolvido por machos e fêmeas na espécie humana, especialmente no que se refere ao cuidado parental, é bastante diferenciado (Keller, 2007). As fêmeas são responsáveis, principalmente, pela gestação e pelos cuidados primários logo após o nascimento, bem como pelas primeiras relações sociais. Os machos, por sua vez, adquirem a função principal de provedores e protetores da família. Esses diferentes papéis demandam o desenvolvimento de diferentes habilidades, as quais são estabelecidas a partir de diferenciações de caráter genético e de caráter cultural, de forma integrada.

Assim, meninos e meninas são predispostos, em geral, a diferentes padrões comportamentais, no sentido de que ficam sob controle de estimulações distintas e reagem diferentemente a determinadas formas de interação. Junto a isto, o desenho bio-sociocultural das relações e brincadeiras infantis favorece, desde a primeira infância, o desenvolvimento sexualmente dimórfico de habilidades (Hansen \& cols., 2007).

Como Silva e cols. (2006) destacam, a identificação com o grupo de pares é fundamental para a construção da própria identidade. Considerando o dimorfismo sexual já discutido, a tendência é de que um indivíduo identifique-se com outros membros do mesmo sexo, em função de preferências e padrões comportamentais similares. Assim, há a expectativa de que indivíduos de um determinado sexo possuam identidade de gênero e papel sexual concordantes. Como se desenvolve, portanto, um indivíduo que apresenta padrões de gênero típicos do sexo oposto?

\section{Padrões Típicos de Gênero e a Gênese da Orientação Sexual}

Apesar de não haver dados estatísticos de prevalência (Associação Americana de Psiquiatria, 2000/2003), vários autores indicam que a existência de número elevado de indivíduos que apresentam padrões de gênero típicos do sexo oposto gerou a classificação recorrente na literatura de pessoas (crianças ou adultos, homens ou mulheres) que não apresentam esses padrões, com diagnóstico clínico ou não, como sendo não conformes de gênero (Fridell \& cols., 2006). Indivíduos classificados como não conformes de gênero teriam, assim, padrões típicos de gênero inversos ao sexo biológico. Contudo, não foi encontrada, ainda, nenhuma pesquisa específica sobre como ocorre a construção da identidade de gênero nesses indivíduos.

Alexander, Wilcox e Woods (2009) afirmam que o interesse por atividades e brinquedos congruentes com o sexo é um fator importante para o desenvolvimento do gênero. Para esses autores, o desenvolvimento sexual do cérebro (a partir de efeitos hormonais pré-natais e neonatais) explicaria as diferenças comportamentais de gênero observadas desde a primeira infância. Ressaltam, ainda, o papel das interações sociais, enquanto socialização de gênero (como segregação sexual, estereotipia etc.), na consolidação da identidade de gênero de cada indivíduo.

Surge aqui a necessidade de reiterar que a identidade de gênero parece resultar não apenas de padrões típicos de gênero, mas da interação dos mesmos com o ambiente social no qual o indivíduo se insere e do papel do grupo social no qual está inserido.

Paul (1993), por exemplo, afirma que indivíduos não conformes de gênero tenderiam a ser segregados, especialmente os meninos (e.g., Hansen \& cols., 2007; Silva \& cols., 2004), incorrendo em uma precoce observação da própria sexualidade. Ao investigar crianças com diagnóstico de transtorno de identidade sexual, Cohen-Kettenis, Owen, Kaijser, Bradley e Zucker (2003) descrevem as implicações da não conformidade de gênero, especialmente nas relações sociais entre pares, sendo os meninos os mais atingidos. Segundo esses autores, tais diferenças comportamentais resultariam em relações sociais empobrecidas e, consequentemente, em incidência mais elevada de problemas comportamentais.

Esse processo tende a ocorrer em um período onde a interação com os pares, especialmente através da brincadeira, adquire papel fundamental para o desenvolvimento da identidade e, consequentemente, da identidade de gênero (e.g., Harris, 1999; Silva, 2006).

A não conformidade de gênero tem sido repetidamente correlacionada à homossexualidade, com significância elevada, mas não total. Lippa (2002) afirma que essa correlação entre meninos corresponde a Cohen's $d$ D 1:31, e entre meninas, a $d$ D 0:96 (para outros dados estatísticos, ver Bailey, Dunne \& Martin, 2000; Cohen, 2002; Pillard \& Weinrich, 
1986; Purcell, Blanchard \& Zucker, 2000), inclusive em sociedades não ocidentais (Paul, 1993). Para Bem (1996), as diferenças entre o que define o masculino e o feminino teriam uma base biológica (o que tem sido reiteradamente corroborado pela literatura, como já apresentado neste trabalho), tendendo a ocorrer precocemente. Tais diferenças, em uma comunidade socialmente estruturada a partir da divisão sexual, teriam um importante papel no desenvolvimento relacional e sexual posterior.

Kraemer e cols., (2009) encontraram efeitos significativos, por meio da análise de variância, entre produtos da ação hormonal (no caso, medida 2D:4D) e padrões de gênero, mas não com a orientação sexual. Revendo a literatura, esses autores afirmam, ainda, que em mulheres a correlação entre hormônios e orientação sexual só ocorre quando há não conformidade de gênero. Savin-Williams e Diamond (2000) fazem uma revisão da literatura e afirmam que em muitos aspectos comportamentais, as diferenças de gênero são superiores às resultantes da orientação sexual, havendo maior correlação interna em grupos divididos de acordo com o sexo do que pela orientação sexual. Esses autores discutem, ainda, que entre homossexuais serão encontradas diferentes trajetórias de gênero (tanto conformes quanto não conformes) não podendo ser atribuída uma relação direta entre gênero e orientação sexual.

Esses dados podem ser interpretados como indicativos que a relação entre orientação sexual e hormônios é indireta, sendo mediada pelos padrões de gênero. De acordo com a teoria de Bem (1996), seria esperado que essa relação fosse mais forte em indivíduos do sexo masculino, já que a influência dos pares seria mais intensa (com padrões de rejeição de indivíduos não conforme de gênero), do que em indivíduos do sexo feminino.

Udry e Chantala (2006) discutem as diferentes teorias existentes sobre os determinantes da orientação sexual, havendo duas correntes principais - uma de cunho biológico e outra, social. Essa mesma divisão é proposta por Lippa (2002). Ambos os artigos destacam, dentre as teorias biológicas, a hipótese de influência hormonal prénatal sobre o desenvolvimento do sistema nervoso central. Os autores defendem as teorias biológicas em função da relação entre padrões de gênero e orientação sexual, propondo que haveria uma explicação comum aos dois, como um elemento causal. Contudo, Udry e Chantala afirmam também que a não conformidade de gênero é preditiva da orientação sexual para homens, mas não para mulheres o que eles explicam com base em mecanismos diferentes para a orientação sexual masculina e feminina. Apesar dos autores classificarem a proposta de Bem (1996) como social, tal proposta considera que haveria uma origem biológica (possivelmente na influência hormonal pré-natal) para os padrões de gênero, mas que a evolução desses para a orientação sexual seria resultante das relações sociais. Tais relações seriam mais segregacionistas de indivíduos não conformes de gênero quando esses fossem do sexo masculino, o que ocasionaria uma maior incidência de orientação homossexual nestes (corroborando os dados obtidos por Udry \& Chantala, 2006).

Silva (2006) defende o papel do vínculo estabelecido entre os pares na construção de uma cultura própria, a qual seria, inclusive, uma cultura de gênero, com normas e valores específicos pautados nos conceitos de masculino e feminino. Como o grupo tende a ser mais importante para meninos, observa-se que a segregação daqueles que não se adequam às regras é maior do que no caso de grupos de meninas. A autora destaca ainda a intersubjetividade como processo de desenvolvimento de sintonia e reciprocidade na relação interpessoal entre dois ou mais membros de um grupo.

Uma vez que as formas de comportar-se não são homogêneas, mas categorizadas, e na maioria das vezes, de forma oposta (homens e mulheres; adultos e crianças etc.), as crianças precisam então descobrir ou decidir quem são, onde se encaixam, a qual grupo pertencem para a partir dai, trabalhar esta inserção. A criança se compara aos pares e assim, as meninas se tornam mais parecidas com as outras meninas e os meninos com os outros meninos. A maior preocupação de meninos e meninas é ser como, pertencer, não estar em dissonância seja no jeito de vestir, de falar, de comportar-se etc. (Silva, 2006, p. 55)

Geerts (1997) afirma que a convergência interpessoal pode ser um importante fator tanto para a satisfação nas relações interpessoais quanto, inclusive, por ter consequências sobre o desenvolvimento de quadros depressivos no curso da vida. Esta convergência seria, assim, uma importante característica para o desenvolvimento psicossocial saudável e para a criação de vínculo entre pares.

Segundo Bouhuys (2003), o estudo da interação a partir de comportamentos não verbais deve analisar a influência comportamental recíproca, ou seja, de que forma o comportamento de um indivíduo é afetado e afeta o comportamento daquele com quem interage. Para esse autor, tal interação não verbal pode expressar elementos da personalidade do individuo e é importante para o desenvolvimento de atitudes de rejeição ou aproximação (quando há reciprocidade). Assim, a convergência interpessoal seria um fenômeno que ocorreria ao longo da interação, podendo ser definida como a coordenação da interação social a partir da adoção de um mesmo ritmo, de movimentos similares, intensidades e frequências comportamentais parecidas etc. Isto é, as diferenças no modo de se comunicar não verbalmente tendem a diminuir ao longo da interação até um ponto de convergência.

Bouhuys (2003) afirma que a convergência interpessoal tem sido estudada de modo a ter sido identificada a importância da mesma para a atração e para o apego. Dessa forma, na infância, a convergência teria grande importância na relação mãe-bebê e com o tempo seria fundamental para o bom funcionamento e satisfação nas interações sociais com amigos e parceiros amorosos.

Assim, pode-se refinar a pergunta anterior da seguinte forma: como ocorre tal intersubjetividade quando a convergência desenvolve-se com indivíduos que pertenceriam a outro grupo, isto é, indivíduos do sexo oposto, considerandose as normas da cultura de gênero? Ou seja, quais os efeitos da ausência dessa convergência na relação entre pares do mesmo sexo?

Bem (1996) propõe que, dependendo de fatores relativos ao processo de socialização, o desenvolvimento de uma identificação com o sexo oposto em detrimento do próprio sexo poderia levar a um sentimento de estranhamento deste, 
podendo esse sentimento evoluir para uma atração românticoerótica. Assim, a identidade de gênero seria fundamental ainda mais do que simplesmente a adoção de padrões típicos de gênero - para o desenvolvimento da orientação sexual. Isto é, para Bem, a identificação com o próprio sexo estaria relacionada ao estranhamento do sexo oposto e, por conseguinte, à atratividade por ele; da mesma forma que a identificação com o sexo oposto poderia favorecer o estranhamento do mesmo sexo e a decorrente atratividade por ele.

Assim, propõe-se aqui que a atração pelo diferente foi selecionada evolutivamente. Resta, ainda, a necessidade de identificar se a 'diferença' que estaria relacionada à atração seria anatômica ou comportamental." Assim, pode-se propor que haveria sido selecionada a atração sexual pelo diferente. Contudo, volta-se à questão inicial: indivíduos que se identificam com pessoas do sexo oposto tenderiam a se sentir atraídos pelos "diferentes" anatômica ou comportamentalmente? Ou ainda, o que seria fundamental para a seleção dos parceiros românticos - a diferença em si ou a construção de uma identidade sexual diferente?

Essa seleção da atração pelo diferente, sem determinar quais as diferenças específicas, seria suficiente para garantir uma maior probabilidade de atração por indivíduos do outro sexo a partir de um menor custo biológico, já que prescindiria do desenvolvimento de caracteres anátomo-fisiológicos de identificação de padrões masculinos em mulheres e padrões femininos em homens. Ambos os sexos podem ter evoluído com um funcionamento comum, mais basilar, que seria a atração pelo diferente.

O papel da identidade sexual no desenvolvimento da orientação sexual pode ser fundamental no sentido de reduzir o determinismo absoluto entre estereotipia de gênero e orientação sexual - o qual, como afirmado anteriormente, não se sustenta a partir das evidências. Isto é, mesmo que um indivíduo apresente características típicas do sexo oposto, sua identidade com indivíduos do mesmo sexo seria uma variável relevante no estabelecimento de sua orientação sexual. Assim, seria um elo a mais na cadeia de eventos, compreendendo em si as interinfluências biológicas e sociais.

Lippa (2008) investigou se as diferenças já encontradas entre homens e mulheres heterossexuais apareceriam em amostras homossexuais. O autor relata que indivíduos bissexuais (em ambos os sexos) tendem a apresentar padrões comportamentais e resultados em testes similares aos heterossexuais. Entre os participantes homossexuais, por sua vez, foram encontrados resultados similares aos heterossexuais do sexo oposto, especialmente em interesses típicos de cada gênero e na identidade de gênero. Apesar dessa pesquisa ser restrita a indivíduos britânicos, o que restringe a generalidade dos dados, ela se configura como um indício preliminar da relação entre esses padrões, a identidade de gênero e a orientação sexual.

Tendo por base a proposição de quatro "porquês" para a explicação de um dado comportamento (Timbergen, 1966/1965), pode-se sintetizar a presente argumentação da seguinte forma:

Causas imediatas. Seriam causas imediatas as alterações fisiológicas resultantes das interações sociais, especialmente na segunda infância. A cada interação, o organismo produziria alterações, especialmente hormonais, que favoreceriam o desenvolvimento da atratividade sexual por indivíduos do grupo de padrões de gênero típicos da identidade oposta à sua. Dessa forma, o principal para eliciar tais alterações hormonais não seria se o outro indivíduo é do mesmo sexo ou do sexo oposto, anatomicamente falando, mas sim a que grupos de gênero tais indivíduos pertencem e com os quais se identificam.

Ontogênese. A construção da identidade seria variável de acordo com cada grupo social específico e de experiências pessoais de integração, identificação e segregação social. A cultura tenderia a organizar os grupos sociais a partir de regras de conduta sexual estritamente definidas e considerando apenas duas classificações centrais - feminino e masculino. Tal dicotomização das relações sociais seria não só fortalecida por diversas práticas culturais como seria grande responsável pela segregação de indivíduos que não se enquadram nesse modelo, proporcionando a criação de grupos socialmente excluídos que tenderiam a ter maior interação entre si. Dessa forma, não haveria uma simples inversão de gênero, mas a partir da construção da identidade de gênero de modo não conforme, a criação de outra identidade específica àquele grupo em particular.

Valor adaptativo. A convergência e a estranheza teriam papel fundamental, do ponto de vista biológico, para o desenvolvimento da identificação e a emissão de comportamentos respondentes de excitação sexual na presença de um determinado indivíduo, a partir da produção de hormônios específicos. $\mathrm{O}$ valor adaptativo desses mecanismos consiste, exatamente, na alta probabilidade de formação de grupos a partir do sexo e no interesse pelo sexo oposto, já que a identificação com indivíduos do mesmo sexo seria mais provável e frequente. Tal interesse pelo sexo oposto é um elemento fundamental para a reprodução e, tendo por base essa proposta, teria baixo custo evolutivo (já que seriam mecanismos mais simples). A emergência de padrões atípicos de gênero seria uma das possibilidades desse princípio, com menor probabilidade de ocorrência e, assim, não afetando consideravelmente o valor adaptativo do mecanismo.

Processo evolutivo. A espécie teria evoluído com diferenças comportamentais, além das anatômicas, entre machos e fêmeas, de acordo com as demandas de especialização de papéis sexuais. Haveria, assim, uma tendência de agrupamento a partir das similaridades e de atratividade sexual por indivíduos que possuíssem papel sexual distinto. Tal produto evolutivo garantiria uma probabilidade elevada de relação entre machos e fêmeas sem custo elevado e sem prejudicar a predisposição à formação de vínculos a partir da suscetibilidade ao prazer.

É importante ressaltar que essa proposta não tem a pretensão de compreender o desenvolvimento da orientação sexual como um todo, já que, conforme mencionado no princípio do texto, acredita-se que a orientação sexual é um fenômeno muito mais complexo, não possuindo apenas uma linha de desenvolvimento. Contudo, defende-se (tal qual Bem, 1996) que essa forma de analisar contempla grande parte dos dados existentes hoje na literatura e merece, assim, ser ainda melhor explorada e desenvolvida a partir de pesquisas empíricas. 


\section{Considerações Finais}

A perspectiva evolucionista pode ser um olhar diferenciado para fenômenos já estudados, trazendo como sua maior contribuição a possibilidade de articulação entre a fundamentação biológica e a contextualização sociocultural como elementos indissociáveis para a compreensão de todo e qualquer fenômeno psicológico.

No que se refere à orientação sexual em si, esse olhar se traduz na apropriação de diversos dados já existentes na literatura e na reanálise dos mesmos, de modo a destacar a influência do dimorfismo sexual (empiricamente observado a partir dos padrões típicos de gênero) na construção da identidade sexual a partir das interações sociais e do desenvolvimento da orientação sexual como decorrência desse processo.

Espera-se, assim, poder contribuir com o estudo de outros problemas de pesquisa, a partir da proposição de novos enfoques e metodologias, em especial a partir da retomada do desafio proposto por Tinbergen (1965/1966) de utilizar os "quatro porquês" como diretrizes epistemológicas no estudo do comportamento.

\section{Referências}

Alexander, G. M. (2003). An evolutionary perspective of sextyped toy preferences: Pink, blue, and the brain. Archives of Sexual Behavior, 32, 7-14.

Alexander, G. M., \& Hines, M. (2002). Sex differences in response to children's toys in nonhuman primates (Cercopithecus aethiops sabaeus). Evolution and Human Behavior 23, 467-479.

Alexander, G. M., Wilcox, T., \& Woods, R. (2009). Sex differences in infants' visual interest in toys. Archives of Sexual Behavior, 38, 427-433.

Almeida, C. M. (2007). Descrição do atendimento psicoterápico de uma mulher com hiperplasia adrenal congênita. Trabalho de Conclusão de Curso, Universidade Federal do Pará, Belém.

Associação Americana de Psiquiatria (2003). Manual de diagnóstico e estatística de transtornos mentais (DSM-IV-TR) (C. Dornelles, Trad.). Porto Alegre: Artmed. (Trabalho original publicado em 2000)

Bailey, J. M., Dunne, M. P., \& Martin, N. G. (2000). Genetic and environmental influences on sexual orientation and its correlates in an Australian twin sample. Journal of Personality and Social Psychology, 78, 524-536.

Baker, S. W. (1980). Biological influences on human sex and gender. Signs, 6, 80-96.

Bem, D. J. (1996). Exotic becomes erotic: A developmental theory of sexual orientation. Psychological Review, 103, 320335.

Bem, D. J. (2000). Exotic becomes erotic: Interpreting the biological correlates of sexual orientation. Archives of Sexual Behavior, 29, 531-548.

Birke, L. I. A. (1981). Is homosexuality hormonally determined? Journal of homosexuality, 6, 35-49.

Bouhuys, A. (2003). Ethology and depression. Em F. J. Bremer (Org.), Nonverbal behavior in clinical settings (pp. 233-262). Oxford: Oxford University Press.
Bussab, V.S., \& Ribeiro, F. L. (1998). Biologicamente cultural. Em L. Souza, M. F. Q. de Freitas \& M. M. P. Rodrigues (Orgs.), Psicologia: reflexões (im)pertinentes (pp. 175-193). São Paulo: Casa do Psicólogo.

Cohen, K. M. (2002). Relationship among childhood sexatypical behavior, spatial ability, handedness, and sexual orientation in men. Archives of Sexual Behavior, 31, 129-143.

Cohen-Kettenis, P. T., Owen, A., Kaijseer, V. G., Bradley, S. J., \& Zucker, K. J. (2003). Demographic characteristics, social competence, and behavior problems in children with gender identity disorder: A cross-national, cross-clinic comparative analysis. Journal of Abnormal Child Psychology, 31, 41-53.

Feldman, D. (2003). Puesta a punto bibliográfica sobre la relación de los conceptos parafilias y abuso sexual infantil. Trabalho de Conclusão de Curso, Universidad de Belgrano, Buenos Aires.

Gadpaille, W. J. (1980). Cross-species and cross-cultural contributions to understanding homosexual activity. Archives of General Psychiatry, 37, 349-356.

Gangestad, W. S., Haselton, G. M., \& Buss, M. D. (2006). Evolutionary foundations of cultural variation: Evoked culture and mate preferences. Psychological Inquiry, 2, 75-95.

Geerts, E. A. H. M. (1997). An ethological approach of interpersonal theories of depression. Tese de Doutorado, Rijksuniversiteit Groningen, Gronigen.

Gooren, L. (2006). The biology of human psychosexual differentiation. Hormones and Behavior 50, 589-601.

Hansen, J., Macarini, S. M., Martins, G. D. F., Wanderlind, F. H., \& Vieira, M. L. (2007). O brincar e suas implicações para o desenvolvimento infantil a partir da Psicologia Evolucionista. Revista Brasileira de Crescimento e Desenvolvimento Humano, 17, 133-143.

Harris, J. R. (1999). Diga-me com quem anda ... Rio de Janeiro: Objetiva.

Hassett, J. M., Siebert, E. R., \& Wallen, K. (2008). Sex differences in rhesus monkey toy preferences parallel those of children. Hormones and Behavior, 54, 359-364.

Haussmann, M. (2005). Questão de simetria. Viver: Mente e cérebro, 8, 40-45.

Keller, H. (2007). Culture of infancy. Alemanha: University of Osnabrück.

Kinsey, A. C., Pomeroy, W. B., \& Martin, C. E. (1948). Sexual behavior in the human male. Filadelfia: W. B. Saunders Company.

Kraemer, B., Noll, T., Delsignore, A., Milos, G., Schnyder, U., \& Hepp, U. (2009). Finger length ratio (2D:4D) in adults with gender identity disorder. Archives of Sexual Behavior, 38, 359-363.

Lalumière, M. L., Blanchard, R., \& Zucker, K. J. (2000). Sexual orientation and handedness in men and women: A meta-analysis. Psychological Bulletin, 126, 575-592.

LeVay, S. (1996). Queer science: The use and abuse of research into homosexuality. Massachussets: Massachussets Institute of Tecnology Press.

Lippa, R. A. (2002). Gender-related traits of heterosexual and homosexual men and women. Archives of Sexual Behavior, 31, 83-98.

Lippa, R. A. (2005). Subdomains of gender-related occupational interests: Do they form a cohesive bipolar M-F dimension? Journal of Personality 73, 693-730. 
Lippa, R. A. (2008). Sex differences and sexual orientation differences in personality: Findings from the BBC internet survey. Archives of Sexual Behavior, 37, 173-187.

Lorenz, K. (1986). Evolução e modificação do comportamento (M. B. S. do Nascimento, Trad.). Rio de Janeiro: Interciência. (Trabalho original publicado em 1965)

Menezes, A. B. C., \& Brito, R. C. S. (2007). Reflexões sobre a homossexualidade como subproduto da evolução do prazer. Psicologia em Estudo, 12, 133-139.

Menezes, A. B. C. (2005). Análise da investigação dos determinantes do comportamento homossexual humano. Dissertação de mestrado, Universidade Federal do Pará, Belém.

Minton, H. L., \& McDonald, G. J. (1984). Homosexual identity formation as a developmental process. Em J. P. De Cecco \& D. A. Parker (Eds.), Sex, cells, and same-sex desire: The biology of sexual preference (p. 91-104). New York: The Haworth Press.

Paul, J. P. (1993). Childhood cross-gender behavior and adult homosexuality: The resurgence of biological models of sexuality. Journal of Homosexuality. 24, 41-54.

Pillard, R., \& Weinrich, J. (1986). Evidence for a familial nature of male homosexuality. Archives of General Psychiatry, 43, 808-812.

Pinker, S. (2004). Tabula rasa: A negação contemporânea da natureza humana (L. T. Motta, Trad.). São Paulo: Companhia das Letras. (Trabalho original publicado em (2004)

Purcell, D. W., Blanchard, R., \& Zucker, K. J. (2000). Birth order in a contemporary sample of gay men. Archives of Sexual Behavior, 29, 349-356.

Ricketts, W. (1984). Biological research on homosexuality: Ansell's cow or Occam's razor? Journal of Homosexuality, 10, 65-93.

Sardinha, A. P. A. (2007). Hiperplasia adrenal congênita: descrição de um estudo de caso. Trabalho de Conclusão de Curso, Universidade Federal do Pará, Belém.

Savin-Williams, R. C., \& Diamond, L. M. (2000). Sexual identity trajectories among sexual-minority youths: Gender comparison. Archives of Sexual Behavior, 29, 607-627.
Silva, L. I. C. (2006). Papagaio, pira, peteca e coisas dos gêneros. Dissertação de Mestrado, Universidade Federal do Pará, Belém.

Silva, L. I. C., Pontes, F. A. R., Silva, S. D. B., Magalhães, C. M. C., \& Bichara, I. D. (2006). Diferenças de gêneros nos grupos de brincadeira na rua: a hipótese de aproximação unilateral. Psicologia: Reflexão \& Crítica, 19, 114-121.

Skinner, B. F. (1975). The shaping of phylogenic behavior. Journal of the Experimental Analysis of Behavior, 24, 117-120.

Tinbergen, N. (1966). Comportamento animal (P. de Lemos, Trad.). São Paulo: José Olympio. (Trabalho original publicado em 1965)

Tooby, J. \& Cosmides, L. (2000). Evolutionary psychology: Foundational papers. Cambrige: MIT Press.

Trevisan, J. S. (2002). Devassos no paraíso. Rio de Janeiro: Editora Record.

Udry, J. R., \& Chantala, K. (2006). Masculinity-femininity predicts sexual orientation in men but not in women. Journal of Biosocial Science, 38, 797-809.

Van Wyk, P. H., \& Geist, C. S. (1984). Psychosocial development of heterosexual, bisexual, and homosexual behavior. Archives of Sexual Behavior, 13, 505-544.

Wegesin, D. J. (1998). A neuropsychologic profile of homosexual and heterosexual men and women. Archives of Sexual Behavior, 27, 91-108.
Recebido em 27.01.2009

Primeira decisão editorial em 22.05.2009

Versão final em 08.06.2009

Aceito em 06.07.2009 